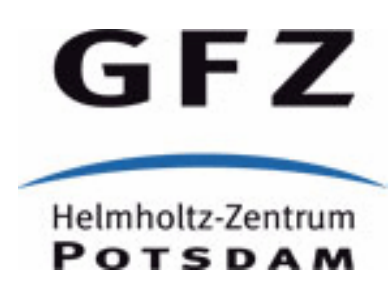

Originally published as:

Blöcher, M.G., Cacace, M., Lewerenz, B., Zimmermann, G. (2010): Three dimensional modelling of fractured and faulted reservoirs: Framework and implementation. - Chemie der Erde - Geochemistry, 70, Suppl. 3, 145-153

DOI: 10.1016/j.chemer.2010.05.014 


\title{
Three dimensional modelling of fractured and faulted reservoirs: Framework and Implementation
}

\author{
M. G. Blöcher ${ }^{\mathrm{a}, \mathrm{b}, *}$, M. Cacace ${ }^{\mathrm{a}, \mathrm{c}}$, B. Lewerenz ${ }^{\mathrm{a}}$, G. Zimmermann ${ }^{\mathrm{a}}$ \\ ${ }^{a}$ Helmholtz Centre Potsdam - GFZ German Research Centre for Geosciences, Telegrafenberg, D-14473 Potsdam, \\ Germany \\ ${ }^{b}$ Brandenburg University of Technology, Konrad-Wachsmann-Allee 1, D-03046 Cottbus, Germany \\ ${ }^{c}$ University of Potsdam, Am Neuen Palais 10, D-14469 Potsdam, Germany
}

\begin{abstract}
Modelling of coupled physical processes in fractured and faulted media is a major challenge for the geoscience community. Due to the complexity related to the geometry of real fracture networks and fault systems, modelling studies have been mainly restricted either to two dimensional cases or to simplified orthogonal fracture systems consisting of vertical and horizontal fractures. An approach to generate three dimensional meshes for realistic fault geometries is presented. The method enables representation of faults in an arbitrary incline as two dimensional planes within a three dimensional, stratified porous matrix of a generic geometry. Based on a structural geological model, the method creates three dimensional unstructured tetrahedral meshes. These meshes can be used for finite element and finite volume numerical simulations. A simulation of a coupled fluid flow and heat transport problem for a two layered porous medium cut by two crossing faults is presented to test the reliability of the method.
\end{abstract}

Keywords: fault systems, fractured reservoirs, thermal hydraulic coupling, finite element method, numerical simulation, 3D mesh generator

\section{Introduction}

The objective of this paper is to describe the influence of fractures and faults on fluid flow and transport properties in fractured and faulted reservoirs. In principle, faults may represent preferential pathways for fluids, or can act as a geological barrier. These two options depend essentially on the origin and orientation of the faults in relation to the recent and paleo stress field (Barton et al., 1995; Gudmundsson, 2001; Moeck et al., 2008; Scheck-Wenderoth et al., 2008; Magri, 2010).

In general, fractured reservoirs can be handled in two ways. The reliability of hydraulic properties of fractured reservoirs is connected to the size of a potential representative elementary

\footnotetext{
*Corresponding author

Email addresses: Guido.Bloecher@gfz-potsdam.de, phone:+49 331 288-1414, fax:+49 331 288-1577 (M. G. Blöcher), Mauro.Cacace@gfz-potsdam.de, phone:-1783, fax:-1782 (M. Cacace), Bjoern.Lewerenz@gfz-potsdam.de, phone:-1343, fax:-1349 (B. Lewerenz), Guenter.Zimmermann@gfz-potsdam.de, phone:-1458, fax:-1577 (G. Zimmermann)

URL: http://www.gfz-potsdam.de, http://www.geoen.org (M. G. Blöcher) Preprint submitted to Chemie der Erde/Geochemistry
} 
volume (REV) (Bear, 1972; De Marsily, 1986). The representative elementary volume (REV) is the smallest volume over which a measurement can be made yielding a value representative of the whole. To completely represent fractures and faults in reservoirs a REV is not sufficient. Below the REV, the relevant parameter is not defined and the material must be treated as heterogeneous with a high variability of its properties. Above the REV the material can be considered as a statistically homogeneous and ergodic medium and can be modelled as an "equivalent homogeneous" medium. An overview concerning this problem, commonly referred to as "the scale effect," and the corresponding concepts to model the hydraulic flow is given by Guéguen et al. (1996). The description of fracture models and their characteristic parameters has been achieved with various theoretical approaches. Several methods have been developed to solve the sophisticated problem of transferring the complex structure of natural rocks to adequate, equivalent models. Such methods include the deterministic fracture networks (Kolditz, 1995a,b; David, 1993), fractal fracture networks (Kosakowski, 1996; Acuna and Yortsos, 1995) and stochastic fracture networks (Cacas et al., 1990b,a; Bruel et al., 1994; Wollrath, 1990; Zimmermann et al., 2000).

In all areas of geo-energy research, (e.g. $\mathrm{CO}_{2}$ sequestration and storage, shale gas and geothermal energy) the development of adequate reservoir and deposit models are of primary concern, while studying the dynamic behaviour during reservoir utilisation. Evaluating the response of geological deposits during $\mathrm{CO}_{2}$ sequestration and storage (Ketzin site; e.g. Juhlin et al. (2007)), shale gas extraction (Barnett shale; e.g. Gale et al. (2007)) or geothermal heat recovery (Groß Schönebeck site; e.g. Blöcher et al. (2010)) requires an understanding of the complex three dimensional geometry of the deposits. This geometry is difficult to assess, because the scale of numerical and experimental investigation alters the size of the measured parameters. Upscaling is an ongoing relevant issue (e.g. Lock et al., 2004; Zimmermann et al., 2003; McDermott et al., 2006). In case their size exceeds the correspondent REV, faults and fractures have to be treated as discrete objects in a reservoir model. Therefore, geometric modelling and mesh generation was the subject of several previous studies (Blessent et al., 2009; Kalbacher et al., 2007). We developed a 3D finite element model with unstructured tetrahedral meshes for matrix properties with embedded 2D discrete surfaces representing the faults and fractures.

The paper is organised as follows: After describing the general modelling techniques applying 3D geological structures and 2D planar surfaces to obtain a combined 3D mesh, we give an example of such a system with two geological layers and two dipping fault systems. This mesh is then used for a coupled thermal-hydraulic simulation. Finally, the results of this model are presented and discussed.

\section{Description of model techniques and methods}

Understanding and predicting physical processes occurring in complex fractured geological systems requires numerical models capable of simulating the coupling between the processes involved in their realistic three dimensional geological framework. The present paper describes a direct approach to generate unstructured tetrahedral meshes suitable for finite element or finite volume numerical simulations of coupled processes for complex faulted natural geological systems. The procedure is fully automated in a $\mathrm{C}++$ source code written by the authors and provides 3D meshes that can be directly imported by existing numerical software. In the following, the different steps of the method are schematically illustrated for a relatively simple case geometry consisting of two geological layers cut by a system of two intersecting faults. 
The first step is to integrate the geological structures defining the geometry of the unstructured model (Figure 1). The input data required are files of scattered data points ( $x-y-z$ coordinates) of the layer interfaces (Figure 1a). The scattered data are read and then interpolated to triangular surfaces in 3D space. For this purpose, we used an algorithm which combines a two-dimensional Delaunay triangulator to calculate $x-y$ values (Shewchuk, 2002) and inverse distance weighting (IDW) interpolator to calculate z-values, see Figure 1b.

In the second stage, faults are implemented in the model (Figure 2). Faults are represented as two dimensional planar structures embedded in a three dimensional geological boundary volume. A multiple regression of the scattered data points describing the geometry of the fault (Figure 2a) is performed to find the best fitting plane of the set of points. The theory behind the multiple regression algorithm can be found in Rinne (2008). After projecting the points onto the plane, the convex hull describing the outer geometry of the fault plane is calculated based on a modified version of Graham's algorithm (Graham, 1972) as described in O'Rourke (1998), see Figure 2b.

Locations of the intersections between the geological surfaces and the convex hulls defining the faults are then calculated (Figure 3a). To represent the trace of the fault in the face of each geological surface, the location of the nodal points of the nearest triangles to the intersection segment is shifted to exactly match the line describing the trace of the fault (Figure 3b).

The final three dimensional Delaunay mesh is generated using the TetGen ${ }^{1}$ program developed at the Weierstraß Institute for Applied Analysis and Stochastic (WIAS) (Si, 2008). TetGen generates adaptive tetrahedral meshes suitable for numerical methods, such as finite element or finite volume methods. For meshing purposes, all three dimensional domains must be defined by their boundaries by means of surface meshes resulting in piecewise linear complexes (PLC), see Figure 4. In order to represent the fault planes as PLCs intersecting facets during the meshing process, crossing of faces has to be avoided. For this purpose, calculated intersection segments between the geological surfaces and the fault planes are added to the fault polygons.

Figure 5a and Figure 5b illustrate the final three dimensional mesh generated by the TetGen program. A constrained Delaunay triangulation along the fault plane is imposed to represent the geometry of the fault facets (Figure $5 \mathrm{c}$ ).

A case-study based on this geometry for a thermo-hydraulic (T-H) problem is presented in the remainder of this paper. For modelling, the numerical simulator OpenGeoSys ${ }^{2}$ is used (Wang et al., 2009; Watanabe et al., 2010). OpenGeoSys is an open source finite element simulator used for the solving of thermal, hydraulic, mechanical and chemical (T-H-M-C) processes in fractured porous media, developed in cooperation between the Department of Environmental Informatics of the Helmholtz Centre for Environmental Research (UFZ, Leipzig), the TU Dresden, the Federal Institute for geosciences and natural Resources (BGR, Hannover), the Paul-Scherrer Institute (PSI, Villigen, Switzerland), the University of Kiel and the University of Edinburgh. TetGen output files are exported to OpenGeoSys format by using the pre-processing software GINA4 $4^{3}$.

\footnotetext{
${ }^{1}$ http://tetgen.berlios.de/

${ }^{2}$ www.opengeosys.net

${ }^{3}$ GINA Version 2.1.5 A Pre- and Postprocessing Tool for the Scientific Program System OpenGeoSys, Copyright 2005-2009, Herbert Kunz, Stilleweg 2, 30655 Hannover, Germany, herbert.kunz@ bgr.de
} 


\section{Description of the sample model}

\subsection{Model geometry}

To show the applicability of the previously generated finite element mesh, a three dimensional $\mathrm{T}-\mathrm{H}$ simulation was performed. The model volume consists of two sub-horizontal geological layers, including two dipping faults (Figure 6). The horizontal north-south and east-west extensions are $200 \mathrm{~m}$, resulting in a horizontal model area of $40.000 \mathrm{~m}^{2}$. The two geological layers are vertically bordered by three curved surfaces. The elevation of top, middle and bottom surface is $55 \mathrm{~m} \pm 5 \mathrm{~m}, 0 \mathrm{~m} \pm 7 \mathrm{~m}$ and $-45 \mathrm{~m} \pm 5 \mathrm{~m}$, respectively. Therefore, an average thickness of $55 \mathrm{~m}$ for layer 1 and $45 \mathrm{~m}$ for layer 2 is established (Table 1).

Both faults are penetrating the two geological layers. Fault 1 has a length of $233 \mathrm{~m}$ and is striking North-East, with dip coordinates of $316.7^{\circ} ; 80.6^{\circ}$. Fault 2 has a length of $184 \mathrm{~m}$ and is oriented perpendicular to fault 1 , having dip coordinates of $225^{\circ} ; 63.2^{\circ}$ (Table 2 ).

\subsection{Initial and boundary conditions}

During the simulation, a general flow field from the South to the North is generated. For this purpose, Dirichlet (or first-type) boundary conditions for pressure are set along the southern and northern boundaries. According to the definition of hydrostatic pressure, the pressure at the southern border is constant at $\mathrm{p}(\mathrm{x}, \mathrm{y}=-100 \mathrm{~m}, \mathrm{z})=\rho \mathrm{gz}+1.75 \mathrm{E}+06 \mathrm{~Pa}$ and at the northern border at $\mathrm{p}(\mathrm{x}, \mathrm{y}=100 \mathrm{~m}, \mathrm{z})=\rho \mathrm{gz}+1.25 \mathrm{E}+06 \mathrm{~Pa}($ Figure $7 \mathrm{a})$, where $\rho\left[1000 \mathrm{~kg} / \mathrm{m}^{3}\right], \mathrm{g}\left[9.81 \mathrm{~m} / \mathrm{s}^{2}\right]$ and $\mathrm{z}$ denotes the fluid density, gravitational acceleration and height of liquid column, respectively. An average hydraulic gradient $\nabla \mathrm{h}=5 \mathrm{E}+05 \mathrm{~Pa} / 200 \mathrm{~m}=0.25$ from the South to the North is provided. For the remaining domain, a pressure value of $1.75 \mathrm{E}+06 \mathrm{~Pa}$ is initialized.

To generate an inflow of hot and cold water from the southern border, Dirichlet boundary conditions for temperature are also applied. Along the southern border, temperature increases from $40^{\circ} \mathrm{C}$ to $80^{\circ} \mathrm{C}$, in going from West to the East resulting in a temperature profile of $\mathrm{T}$ ( $\mathrm{x}, \mathrm{y}=$ $-100 \mathrm{~m}, \mathrm{z})=0.2^{\circ} \mathrm{C} / \mathrm{m} * \mathrm{x}+60^{\circ} \mathrm{C}$ (Figure $7 \mathrm{~b}$ ). For the remaining domain, the initial temperature is set to $60^{\circ} \mathrm{C}$.

\subsection{Parametrisation}

To assure a variation of the hydraulic properties, the upper geological layer was modelled twice as conductive as the lower layer (Table 1). The permeability $k$ of layer 1 is set to $2 \mathrm{E}-14 \mathrm{~m}^{2}$ and the porosity $\phi$ to 0.15 . For layer 2 the permeability $\mathrm{k}$ is set to $1 \mathrm{E}-14 \mathrm{~m}^{2}$ and the porosity $\phi$ to 0.08 . The storage of both layers is derived from the bulk compressibility $\beta[1 / \mathrm{Pa}]$ of the rock and the embedded fluid. Assuming fissured rocks, the storage is set to $7 \mathrm{E}-101 / \mathrm{Pa}$.

The hydraulic properties of the faults vary as well (Table 2). The permeability of fault 1 is set to $1 \mathrm{E}-08 \mathrm{~m}^{2}$ and that of fault 2 to $5 \mathrm{E}-09 \mathrm{~m}^{2}$. The fault transmissivity is defined as the product of the fault permeability $k$ and aperture $a$. To ensure a high contrast between fault transmissivity and matrix conductivity, the aperture of both faults is set to $0.05 \mathrm{~m}$. To provide free fluid flow in the faults, a porosity value of 1.0 is chosen. The storage in the faults is due to the fluid compressibility only and $\beta=4.6 \mathrm{E}-101 / \mathrm{Pa}$ is assigned.

To observe the most significant changes of the temperature field a simulation time of 145 years is chosen. 


\section{Results}

The results are presented in two parts. The first part displays the primary results for fluid pressure, velocity and temperature field of the total domain. The second part will show the pressure, velocity and temperature evolution through time of three observation points within the faults. After starting the simulation, the pressure and velocity field of the model domain change due to the chosen boundary conditions. After approximately one month a steady state for pressure and velocity field is achieved (Figure 8).

Due to the fact that the implemented faults do not cut the southern and northern borders of the model, matrix flow is predominant in these areas. Therefore, the highest pressure gradients are at the northern and southern borders of the model (Figure 8a). In proximity to the cutting faults, the isobars (surfaces of constant pressure) are sub-horizontal due to high flow rates within the faults. Maximum Darcy velocities of $\mathrm{v}=1 \mathrm{E}-04 \mathrm{~m} / \mathrm{s}$ can be observed inside the faults (Figure $8 b$ ). Despite low pressure gradients, high flow rates occur in the fault planes. High values of fluid velocity are the result of the relative high transmissivity of the faults with respects to the surrounding domain.

Figure $8 \mathrm{~b}$ shows the stationary flow field. As described above, highest flow velocities can be observed in the fault planes. The applied pressure boundaries force a regional flow field from the South to the North. The average velocity at the southern and northern regions is $1 \mathrm{E}-07 \mathrm{~m} / \mathrm{s}$, with maximum inflow to the faults from the South. In the rest of the domain, outflow from the faults into the rock matrix is pronounced. In the central part of the model, faults act as the predominant flow paths. In contrast, low velocities (less than $1 \mathrm{E}-08 \mathrm{~m} / \mathrm{s}$ ) characterize the eastern and western boundaries. An additional important fact is that at the southern edge of fault 1 and fault 2, backward flow from the North to the South occurs. Pressure equalisation within the faults results in higher matrix pressure at this area. This causes drainage of the rock matrix by the fault system.

Figure $9 \mathrm{a}-9 \mathrm{~d}$ shows the $45^{\circ} \mathrm{C}, 55^{\circ} \mathrm{C}, 65^{\circ} \mathrm{C}$ and $75^{\circ} \mathrm{C}$ contours at four different time stages.

Before stationary field conditions for pressure and velocity are reached, conductive heat transfer does not affect the initial temperature field significantly (Figure 9a). After achieving the stationary pressure and velocity field, convective heat transfer (advection and diffusion) becomes predominant. The cold water front $\left(\mathrm{T}=55^{\circ} \mathrm{C}\right)$ enters fault 1 after approx. 4 months (Figure $9 \mathrm{~b}$ ). Due to the geometry of fault 1 with respects to the southern boundary of the domain, cold water enters fault 1 in the upper part. After 35 years, (Figure 9c) cold water from fault 1 and hot water from fault 2 are mixed at the fault intersection. The final temperature field (Figure 9d) shows an average temperature of $\mathrm{T}=55^{\circ} \mathrm{C}$ in the northern part which is less than the mean initial temperature of $60^{\circ} \mathrm{C}$. The depression from the mean value is caused because fault 1 is more conductive than fault 2, which drives higher amounts of cold water into the system.

For a detailed observation of the pressure, velocity and temperature evolution inside the two faults, three observation points were set (Figure 10a).

All three observation points are located at the interface between the two geological layers. Observation point 1 is located at the edge of fault 1 and has an elevation of $5.12 \mathrm{~m}$. Observation point 2 is located at the edge of fault 2 and has an elevation of $-3.18 \mathrm{~m}$. Observation point three is located at the intersection of both faults with an elevation of $0.0 \mathrm{~m}$. After starting the simulation the pressure increases at all observation points (Figure 10b). As shown for observation point 3 (Figure 10c), the initial magnitude of the velocity is due to vertical flow only. The observed downward flow is forced by the initial pressure conditions in combination with the chosen pressure boundary. Therefore, an initial increase of fluid pressure is observed. After 1 
month, a stationary pressure and velocity field is reached, as indicated by the horizontal lines in Figure 10b-10c. Differences between the three observation points are due to their specific elevation.

The vertical component of velocity decreases over time from $3 \mathrm{E}-02 \mathrm{~m} / \mathrm{s}$ to $1 \mathrm{E}-08 \mathrm{~m} / \mathrm{s}$, and the horizontal flow from the South to the North with velocities between $1 \mathrm{E}-05 \mathrm{~m} / \mathrm{s}$ and $1 \mathrm{E}-04 \mathrm{~m} / \mathrm{s}$ becomes dominant. As mentioned before, the cold water reaches the fault system at the edge of fault 1 (Figure 10d) after approx. 4 month. After an additional 17 months, cooling at observation point 3 begins. At the same time, hot water reaches fault 2 first. Due to the lower transmissivity of fault 2 , the hot water reaches the intersection point after 10 years, and cooling at observation point 3 stops. Higher amounts of cold water enter the fault intersection (observation point 3 ) from the more conductive fault 1 , causing temperature to decrease to $55^{\circ} \mathrm{C}$. This corroborates the observation of the temperature field for the total domain.

\section{Discussion}

Potential fluid flow along faults depends on the current stress regime of the reservoir. For a normal faulting stress regime, faults have high shear stress and high slip tendency. At the transition of normal to strike slip faulting, the potential fluid flow along these critically stressed faults increases. For strike slip faulting, a reactivation of faults with high slip tendency is possible (Moeck et al., 2008). Currently, faults are represented by planar polygons in 3D space with constant properties for aperture and permeability. Further, the presented sample model does not integrate a mechanical coupling. This restriction was made to keep the sample model simple and is not due to limitations of the applied finite element simulation software. OpenGeoSys provides the possibility of a mechanical coupling, and the hydraulic properties of discrete features can be adjusted in space and time depending on the stress state. These important dependencies of fracture and fault transmissivity can be mapped and modelled (Warpinski et al., 2008; Walsh et al., 2008) and/or determined by laboratory experiments (e.g. influence of asperity creep on fracture permeability by Cuisiat et al. (2002)). The integration of the functional relation between stress state and fault transmissivity will result in a better approximation of the natural systems.

\section{Conclusions}

The presented modelling techniques, methods and the case-study describe the technical workflow from scattered structure geological data to the final finite element simulation. These techniques and methods can be applied for fractured porous media, including fault systems. Since the complexity of the geometric system increases rapidly with increasing numbers of fracture and faults, the applied techniques and methods must be tested by means of future applications including more discrete features than in the case-study. Geo-energy research related topics, e.g. $\mathrm{CO}_{2}$ storage, shale gas extraction and geothermal heat recovery can be benefit from these techniques and methods. The advantage is that dipping structures can be integrated into a 3D body representing a porous media, and interaction between discrete flow paths and rock matrix can be simulated. Further, the complete workflow is captured by open-source software. The integration of discontinuities within the geological layers by normal, reverse, and listric faults (curved normal faults) is ongoing work. 


\section{Acknowledgments}

The authors would like to acknowledge Inga Moeck for fruitful discussions about EarthVision export formats as well as Hang Si and Norihiro Watanabe for the behind-the-scene support of numerous technical questions concerning TetGen and OpenGeoSys, respectively. The constructive comments from O. Kolditz and F. Magri helped to significantly improve the clarity of this contribution: sincere thanks are extended to them both.

\section{References}

Acuna, J., Yortsos, Y., 1995. Application of fractal geometry to the study of networks of fractures and their pressure transient. Water Resour. Res. 31 (3), 527-540.

Barton, C. A., Zoback, M. D., Moos, D., 1995. Fluid flow along potentially active faults in crystalline rock. Geology $23(8), 683-686$.

Bear, J., 1972. Dynamics of Fluids in Porous Media. American Elsevier, New York.

Blessent, D., Therrien, R., MacQuarrie, K., 2009. Coupling geological and numerical models to simulate groundwater flow and contaminant transport in fractured media. Computers \& Geosciences 35 (9), 1897-1906.

Blöcher, M. G., Zimmermann, G., Moeck, I., Brandt, W., Hassanzadegan, A., Magri, F., 2010. 3D numerical modeling of hydrothermal processes during the lifetime of a deep geothermal reservoir. Geofluids.

Bruel, D., Cacas, M., Ledoux, E., de Marsily, G., 1994. Modelling storage behaviour in a fractured rock mass. Journal of Hydrology 162 (3-4), 267-278.

Cacas, M. C., Ledoux, E., de Marsily, G., Barbreau, A., Calmels, P., Gaillard, B., Margritta, R., 1990a. Modeling fracture flow with a stochastic discrete fracture network: Calibration and validation 2. the transport model. Water Resources Research 26(3), 491-500

Cacas, M. C., Ledoux, E., de Marsily, G., Tillie, B., Barbreau, A., Durand, E., Feuga, B., Peaudecerf, P., 1990b. Modeling fracture flow with a stochastic discrete fracture network: Calibration and validation 1. the flow model. Water Resources Research 26(3), 479-489.

Cuisiat, F., Grande, L., Høeg, K., 2002. Laboratory testing of long term fracture permeability in shales. SPE/ISRM Rock Mechanics Conference, 20-23 October 2002, Irving, Texas, 10.

David, C., 1993. Geometry of flow paths for fluid transport in rocks. J. Geophys. Res. 98 (B7), 12267-12278.

De Marsily, G., 1986. Quantitative Hydrogeology. Academic Press, Inc.,Orlando, FL.

Gale, J. F. W., Reed, R. M., Holder, J., 2007. Natural fractures in the Barnett Shale and their importance for hydraulic fracture treatments. AAPG Bulletin 91(4), 603-622.

Graham, R. L., 1972. An efficient algorithm for determining the convex hull of a finite planar set. Information Processing Letters 1 (4), 132-133.

Gudmundsson, A., 2001. Fluid overpressure and flow in fault zones: field measurements and models. Tectonophysics $336(1-4), 183-197$.

Guéguen, Y., Gavrilenko, P., LeRavalec, M., 1996. Scales of rock permeability. Surveys in Geophysics 17, $245-263$.

Juhlin, C., Giese, R., Zinck-Jørgensen, K., Cosma, C., Kazemeini, S. H., Juhojuntti, N., Lüth, S., Norden, B., Förster, A., 2007. 3D baseline seismics at Ketzin, Germany: The CO2SINK project. Geophysics 72 (2), B121-B132.

Kalbacher, T., Mettier, R., McDermott, C., Wang, W., Kosakowski, G., Taniguchi, T., Kolditz, O., 2007. Geometric modelling and object-oriented software concepts applied to a heterogeneous fractured network from the Grimsel rock laboratory. Computational Geosciences 11(1), 9-26.

Kolditz, O., 1995a. Modelling flow and heat transfer in fractured rocks: Conceptual model of a 3-D deterministic fracture network. Geothermics 24 (3), 451-470, hot Dry Rock (HDR) Reservoir Modelling Activities within Europe.

Kolditz, O., 1995b. Modelling flow and heat transfer in fractured rocks: dimensional effect of matrix heat diffusion. Geothermics 24 (3), 421-437, hot Dry Rock (HDR) Reservoir Modelling Activities within Europe.

Kosakowski, G., 1996. Simulation von Strömung und Wärmetransport im variszischen Grundgebirge: Vom natürlichen Kluftsystem zum numerischen Gitternetzwerk. VDI Verlag Reihe 7, Nr. 304, 124

Lock, P. A., Jing, X., Zimmerman, R. W., 2004. Comparison of methods for upscaling permeability from the pore scale to the core scale. Journal of Hydraulic Research 42, 3-8.

Magri, F., 2010. Deep geothermal groundwater flow in the Seferihisar-Balçova area, Turkey: results from transient numerical simulations of coupled fluid flow and heat transport processes. Geofluids.

McDermott, C. I., Lodemann, M., Ghergut, I., Tenzer, H., Sauter, M., Kolditz, O., 2006. Investigation of coupled hydraulic - geomechanical processes at the KTB site: pressure-dependent characteristics of a long-term pump test and elastic interpretation using a geomechanical facies model. Geofluids 6 (1), 67-81. 
Moeck, I., Schandelmeier, H., Holl, H. G., 2008. The stress regime in a Rotliegend reservoir of the Northeast German Basin. International Journal of Earth Sciences 98 (7), 1643-1657.

O’Rourke, J., 1998. Computational Geometry in C, 2nd Edition. Cambridge University Press.

Rinne, H., 2008. Taschenbuch der Statistik., 4th Edition. Verlag Harri Deutsch.

Scheck-Wenderoth, M., Krzywiec, P., Maystrenko, Y., Zühlke, R., Froitzheim, N., 2008. Permian to Cretaceous tectonics of Central Europe. In McCann, T. (Ed): Geology of Central Europe. Geological Society Special Publication, London 2, 999-1030.

Shewchuk, J. R., 2002. Delaunay refinement algorithms for triangular mesh generation. Computational Geometry: Theory and applications $22(1-3), 21-74$.

$\mathrm{Si}, \mathrm{H} ., 2$ 2008. Three dimensional boundary conforming Delaunay mesh generation. PhD. Thesis. Ph.D. thesis, Technische Universität Berlin, Institut für Mathematik.

Walsh, R., McDermott, C., Kolditz, O., 2008. Numerical modeling of stress-permeability coupling in rough fractures. Hydrogeology Journal 16, 613-627.

Wang, W., Kosakowski, G., Kolditz, O., 2009. A parallel finite element scheme for thermo-hydro-mechanical (THM) coupled problems in porous media. Computers \& Geosciences 35 (8), 1631-1641.

Warpinski, N., Mayerhofer, M., Vincent, M., Cipolla, C., Lolon, E., 2008. Stimulating unconventional reservoirs: maximizing network growth while optimizing fracture conductivity. SPE Unconventional Reservoirs Conference, 10-12 February 2008, Keystone, Colorado, USA, 19.

Watanabe, N., Wang, W., McDermott, C. I., Taniguchi, T., Kolditz, O., 2010. Uncertainty analysis of thermo-hydromechanical coupled processes in heterogeneous porous media. Computational Mechanics 45(4), 263-280.

Wollrath, J., 1990. Ein Strömungs- und Transportmodell für klüftiges Gestein und Untersuchungen zu homogenen Ersatzsystemen. PhD. Thesis. Ph.D. thesis, Universität Hannover.

Zimmermann, G., Burkhardt, H., Engelhard, L., 2003. Scale dependence of hydraulic parameters in the crystalline rock of the KTB. Pure and Applied Geophysics 160, 1067-1085.

Zimmermann, G., Körner, A., Burkhardt, H., 2000. Hydraulic pathways in the crystalline rock of the KTB. Geophys. J. Int. 142, 4-14.

\section{List of Figures}

1 Workflow of finite element mesh generation: scattered data points resulting from structural geological modelling (1a) and triangulated interfaces of geological lay-

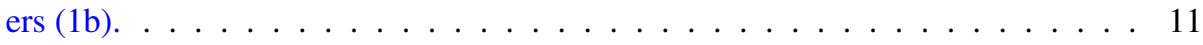

2 Workflow of finite element mesh generation: scattered data points describing the faults geometry (2a) and final convex hull of the outer fault polygons (2b). . . . 12

3 Workflow of finite element mesh generation: calculating faults-layers intersection (3a) and re-arrangement of the nodal position of the triangular elements of the geological surfaces to match the trace of the intersecting fault (3b). . . . . 12

4 Workflow of finite element mesh generation: creating a PLC of the model domain. 13

5 Workflow of finite element mesh generation: final 3D tetrahedral mesh. . . . . . 13

6 Sample model consisting of two geological layers cut by a system of two crossing faults. . . . . . . . . . . . . . . . . . . . . . 14

7 Pressure (7a) and temperature (7b) boundary conditions of the sample model. The pressure boundaries involve a general flow from the South to the North. The temperature boundary generates an inflow of $80^{\circ} \mathrm{C}$ hot water at the south-east and $40^{\circ} \mathrm{C}$ cold water at the south-west corner. . . . . . . . . . . . . 14

8 Simulated steady pressure (8a) and velocity field (8b) achieved after approx. 1 month. . . . . . . . . . . . . . . . . . . . . 15

9 Temperature contour plots $\left(45^{\circ} \mathrm{C}, 55^{\circ} \mathrm{C}, 65^{\circ} \mathrm{C}\right.$ and $75^{\circ} \mathrm{C}$ isosurfaces) at four different time stages. . . . . . . . . . . . . . . . . . . 16

10 Location of three observation points within the fault faces (10a); Simulated pressure (10b) and temperature (10d) values at these observation points and simulated velocity components (10c) at observation point $3 \ldots \ldots \ldots \ldots \ldots$ 


\section{$321 \quad$ List of Tables}

$322 \quad 1$ Geometrical attributes and porous medium properties of geological layers. . . . . 10

з2з 2 Geometrical attributes and medium properties of faults. . . . . . . . . . . . 10 
Table 1: Geometrical attributes and porous medium properties of geological layers.

\begin{tabular}{lccc}
\hline property & unit & layer1 & layer2 \\
\hline \hline average thickness $t$ & {$[\mathrm{~m}]$} & 55 & 45 \\
porosity $\phi$ & {$[-]$} & 0.15 & 0.08 \\
storage $\beta$ & {$[1 / \mathrm{Pa}]$} & $7 \mathrm{E}-10$ & $7 \mathrm{E}-10$ \\
permeability $k$ & {$\left[\mathrm{~m}^{2}\right]$} & $2 \mathrm{E}-14$ & $1 \mathrm{E}-14$ \\
\hline
\end{tabular}

Table 2: Geometrical attributes and medium properties of faults.

\begin{tabular}{lccc}
\hline property & unit & fault1 & fault2 \\
\hline \hline dip direction & {$\left[^{\circ}\right]$} & 316.7 & 225 \\
dip & {$\left[{ }^{\circ}\right]$} & 80.6 & 63.2 \\
length $l$ & {$[\mathrm{~m}]$} & 233.5 & 183.8 \\
aperture $a$ & {$[\mathrm{~m}]$} & .05 & .05 \\
porosity $\phi$ & {$[-]$} & 1 & 1 \\
storage $\beta$ & {$[1 / \mathrm{Pa}]$} & $4.6 \mathrm{E}-10$ & $4.6 \mathrm{E}-10$ \\
permeability $k$ & {$\left[\mathrm{~m}^{2}\right]$} & $1 \mathrm{E}-8$ & $5 \mathrm{E}-9$ \\
\hline
\end{tabular}




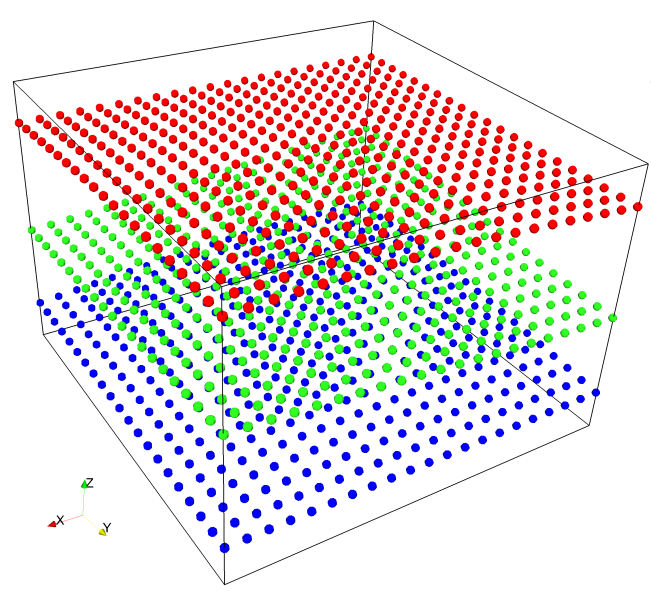

(a)

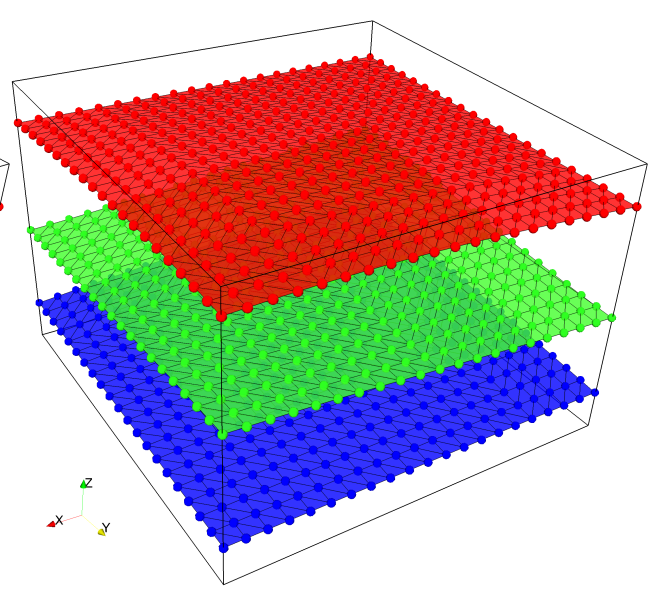

(b)

Figure 1: Workflow of finite element mesh generation: scattered data points resulting from structural geological modelling (1a) and triangulated interfaces of geological layers (1b). 


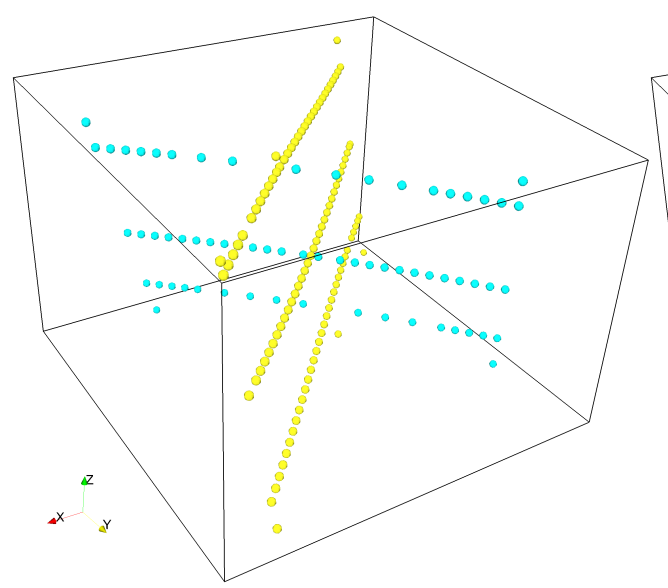

(a)

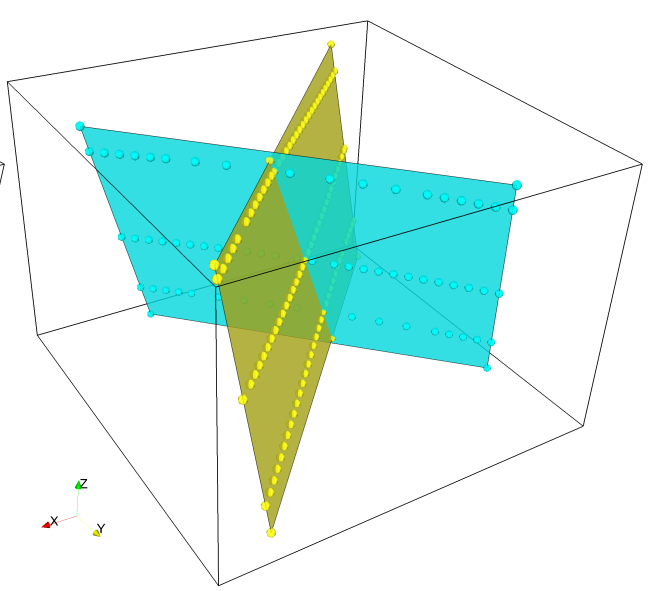

(b)

Figure 2: Workflow of finite element mesh generation: scattered data points describing the faults geometry (2a) and final convex hull of the outer fault polygons (2b).

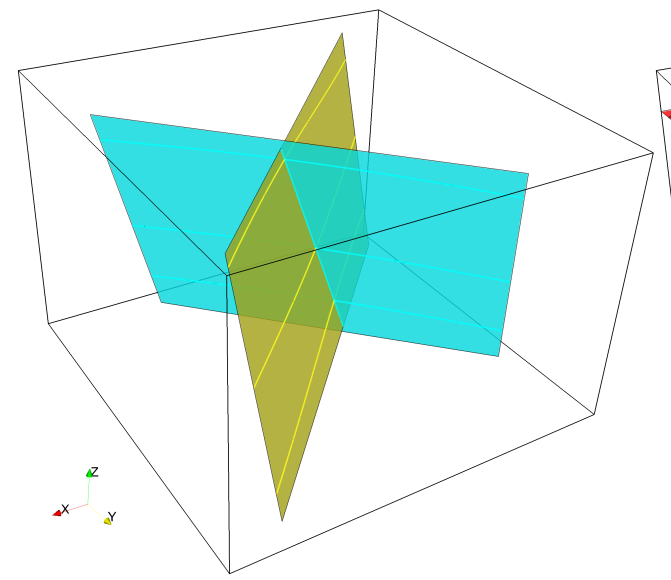

(a)

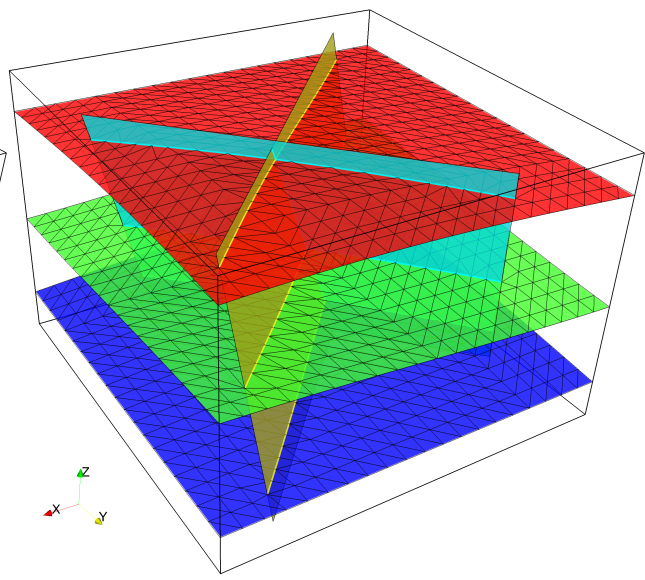

(b)

Figure 3: Workflow of finite element mesh generation: calculating faults-layers intersection (3a) and re-arrangement of the nodal position of the triangular elements of the geological surfaces to match the trace of the intersecting fault (3b). 


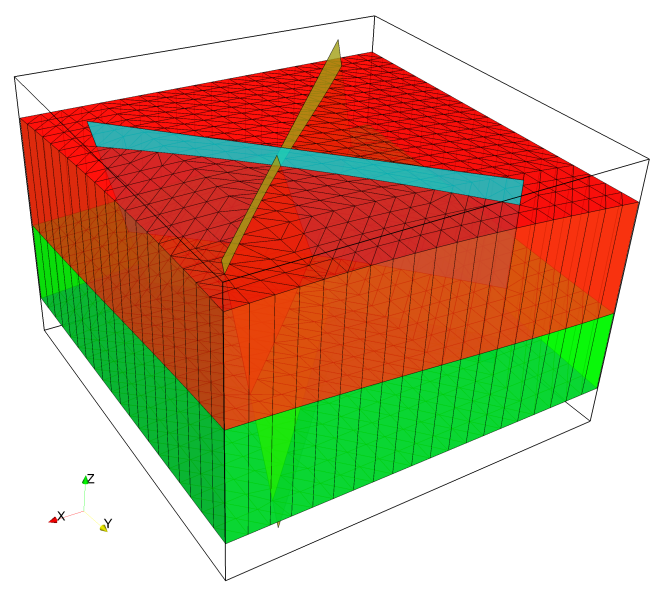

Figure 4: Workflow of finite element mesh generation: creating a PLC of the model domain.

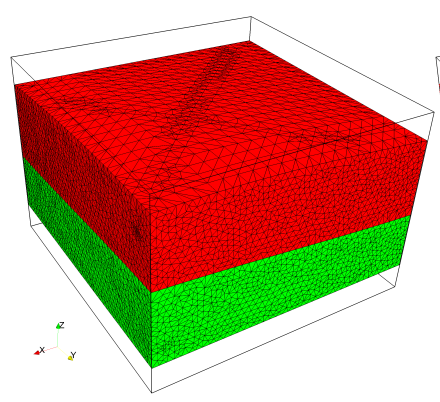

(a)

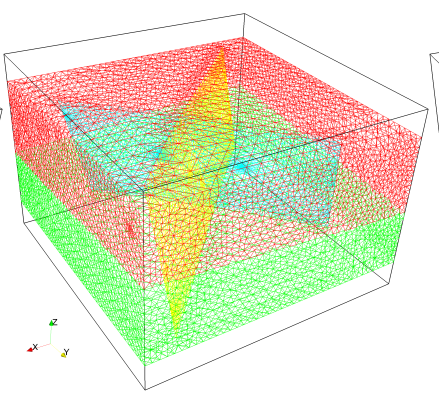

(b)

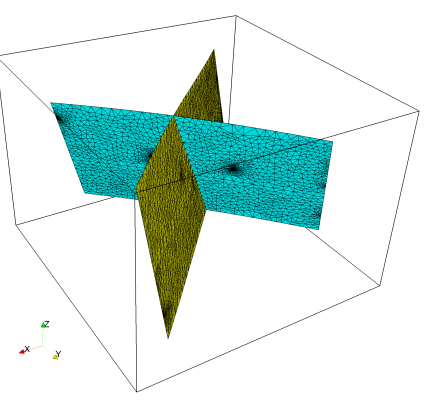

(c)

Figure 5: Workflow of finite element mesh generation: final 3D tetrahedral mesh. 


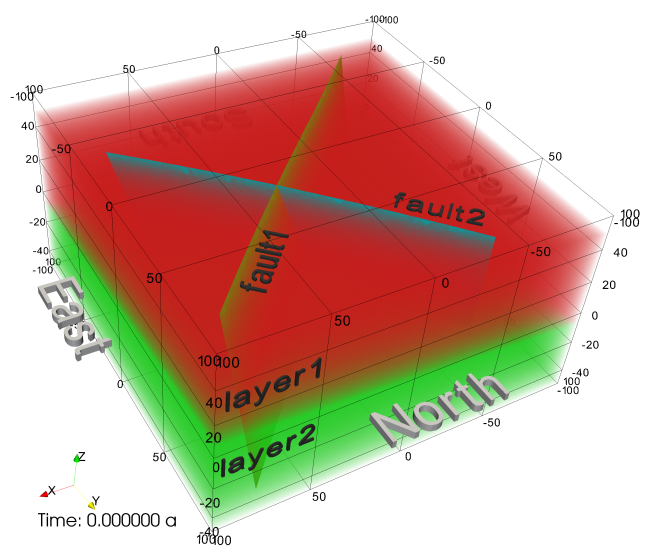

Figure 6: Sample model consisting of two geological layers cut by a system of two crossing faults.

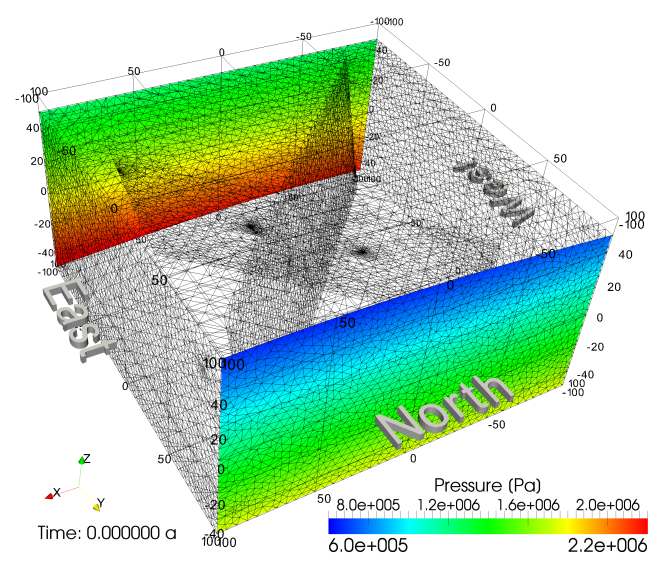

(a)

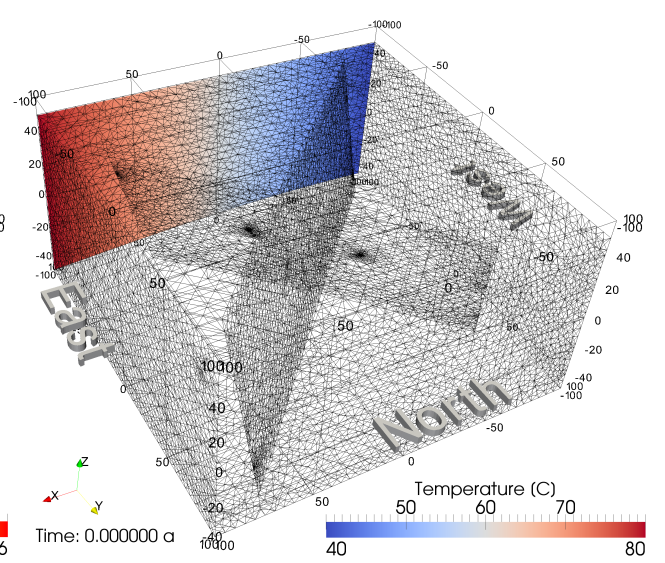

(b)

Figure 7: Pressure (7a) and temperature (7b) boundary conditions of the sample model. The pressure boundaries involve a general flow from the South to the North. The temperature boundary generates an inflow of $80^{\circ} \mathrm{C}$ hot water at the south-east and $40^{\circ} \mathrm{C}$ cold water at the south-west corner. 


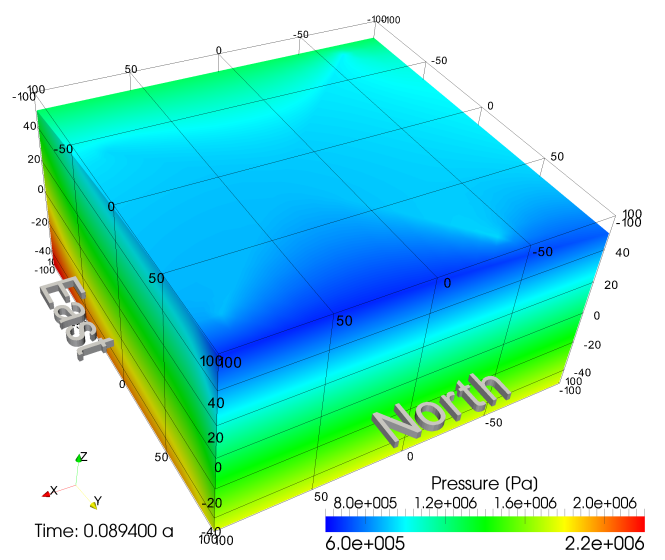

(a)

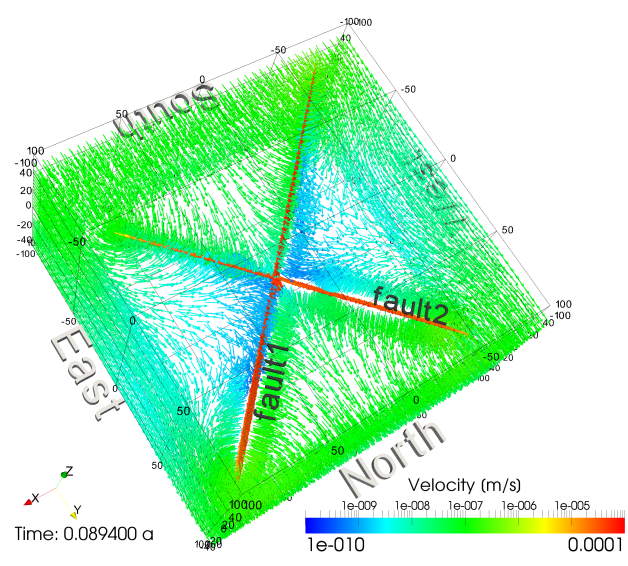

(b)

Figure 8: Simulated steady pressure (8a) and velocity field (8b) achieved after approx. 1 month. 


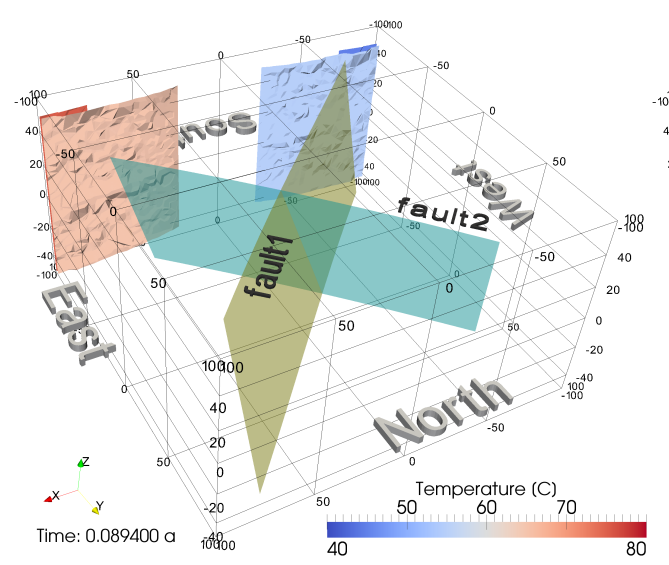

(a)

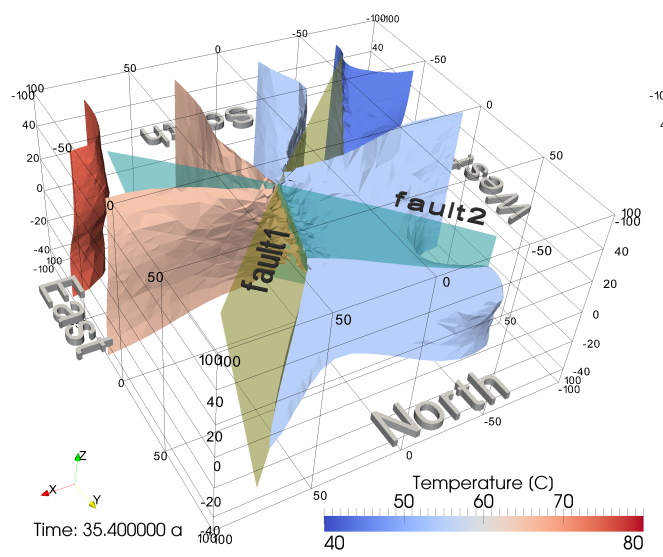

(c)

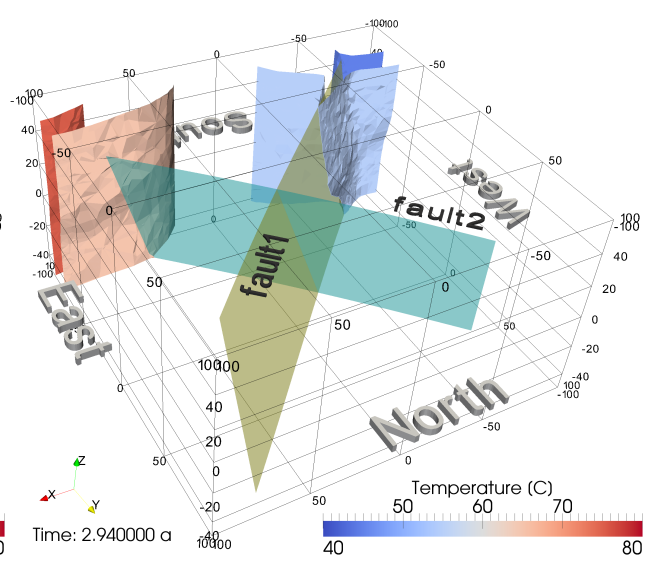

(b)

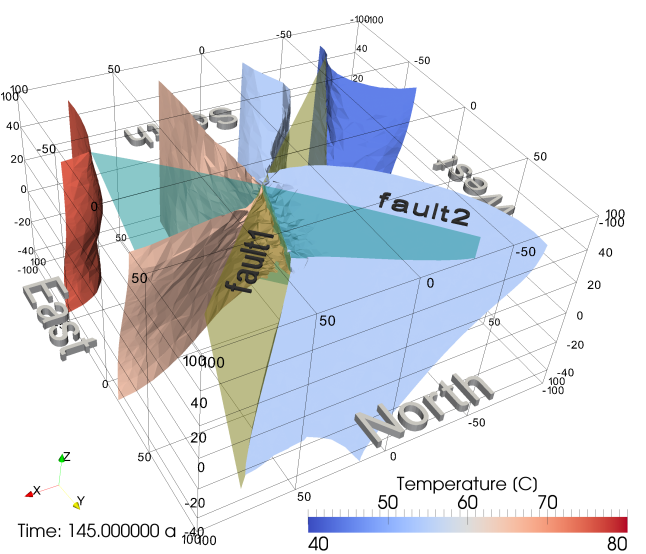

(d)

Figure 9: Temperature contour plots $\left(45^{\circ} \mathrm{C}, 55^{\circ} \mathrm{C}, 65^{\circ} \mathrm{C}\right.$ and $75^{\circ} \mathrm{C}$ isosurfaces) at four different time stages. 


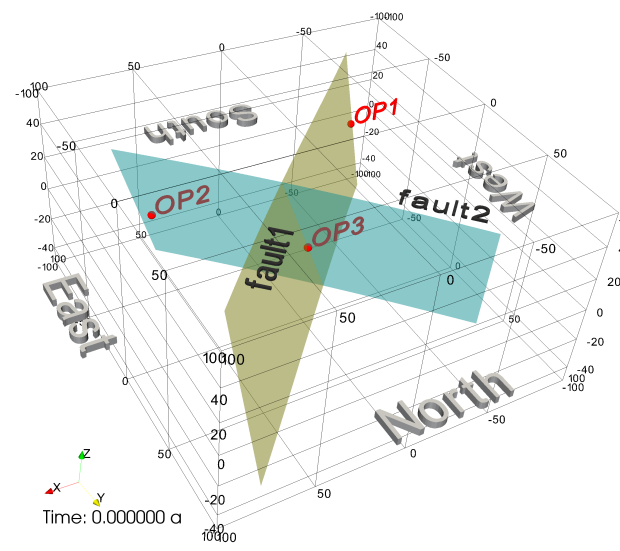

(a)

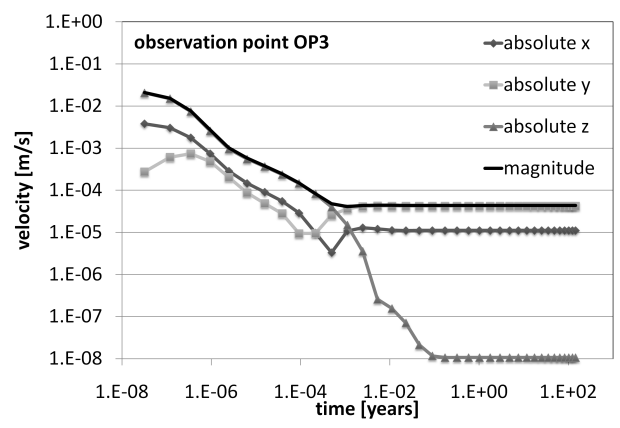

(c)

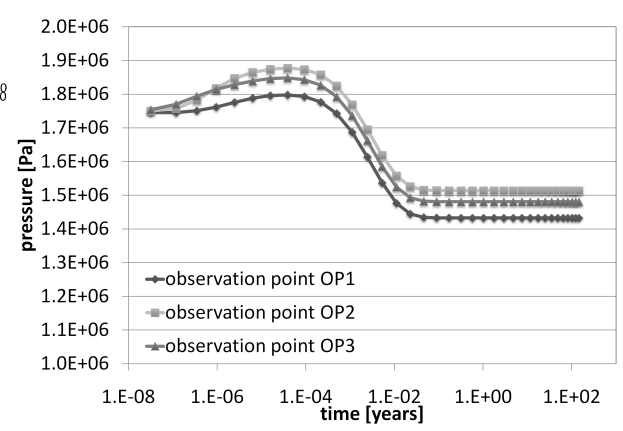

(b)

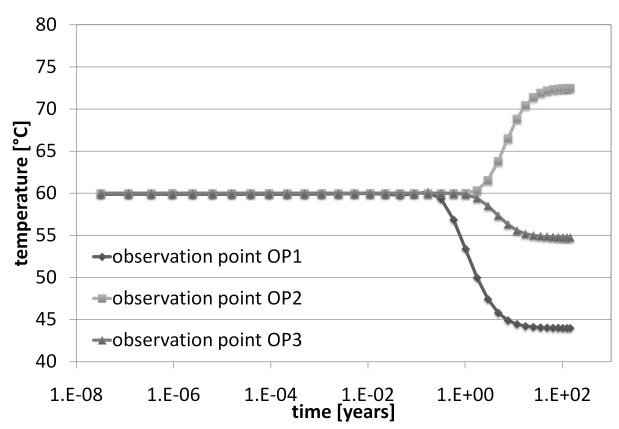

(d)

Figure 10: Location of three observation points within the fault faces (10a); Simulated pressure (10b) and temperature (10d) values at these observation points and simulated velocity components (10c) at observation point 3. 